\title{
Testing Cosmological Models with Negative Pressure
}

\author{
Alberto Cappi ${ }^{1,2}$ \\ ${ }^{1}$ Osservatorio Astronomico di Bologna, via Ranzani 1, I-40127, Bologna, Italy \\ ${ }^{2}$ Observatoire de la Côte d'Azur, B4229,Le Mont-Gros, 06304 Nice Cedex 4, France \\ E-Mail: cappi@astbo3.bo.astro.it
}

Astrophysical Letters and Communications, in press 


\begin{abstract}
There is now strong evidence that the main contribution to the cosmic energy density is not due to matter, but to another component with negative pressure. Its nature is still unknown: it could be the vacuum energy, manifesting itself as a positive cosmological constant with $w \equiv P / \rho c^{2}=-1$ ( $\Lambda$ CDM model), a spatially inhomogeneous and dynamically evolving form of energy with $-1<w<0$ (quintessence, QCDM model), or a dark energy component with $w<-1$, such as a quantized free scalar field (VCDM model). After presenting simple redshift-distance formulae, which are useful for comparing observations with theoretical predictions without numerical integration, I discuss the behaviour of different $w<0$ models in the context of the Alcock-Paczyński geometric test and the statistics of gravitational lensing.
\end{abstract}

Keywords cosmology: theory — cosmology: observations 


\section{Introduction}

The observations of distant Supernovae (Riess et al. 1998, Perlmutter et al. 1999, Riess et al. 2001) indicate that the universe is accelerating, as expected for a positive cosmological constant; in this case it is possible to reconcile a relatively high Hubble constant with an old universe, and a low luminous+dark matter density with the flat geometry implied by the recent CMB observations (de Bernardis et al. 2000). The vacuum energy density can act as a cosmological constant, but its low value and its magnitude comparable to the present matter density represent an unsolved problem (see e.g. Sahni \& Starobinsky 2000, Carroll 2001, and references therein). An intriguing possibility is that the cosmic energy density is dominated by something acting as a variable cosmological "constant" (Ratra \& Peebles 1988); this additional component to the cosmic energy density, now known as "quintessence", would be slowly evolving with time and spatially inhomogeneous, and could be due to a scalar field evolving in a potential coupled to matter through gravitation (Caldwell et al. 1998). Various authors (see Efstathiou 1999, Wang et al. 2000, Balbi et al. 2001) have shown that a flat model including a dominant component of quintessence (with equation of state $w_{Q} \sim-0.65$, see below) is consistent with observations, while Amendola (2000) has put constraints on a quintessence-dark matter coupling.

The observational constraints are still quite uncertain and it is not possible to discriminate between the different model parameters (Podariu \& Ratra, 1999). Moreover, Caldwell (1999) suggested that even models with $w<-1$ satisfy all the observational constraints; he named this hypothetical component "phantom energy", as in his model there is a negative kinetic term in the Lagrangian of the scalar field (see also Schulz \& White 2001). Nevertheless a $w<-1$ equation of state can also be obtained assuming that the vacuum energy is due to a quantized free scalar field of low mass (VCDM model, Parker \& Raval 2001 and references therein).

Changing the equation of state of the dominant energy component of the universe affects the distance-redshift relation and all the distance-dependent physical quantities. The effects become important in deep redshift surveys. Unfortunately, in most cases it is not possible to find exact analytical expressions for the distanceredshift relation (e.g. McVittie G.C. 1965). Models with a cosmological constant are now well studied, and distances and other useful quantities have been obtained through numerical integration (Refsdal, Stabell \& de Lange 1967) or using elliptic integrals (Feige 1992). An excellent approximation for $\Lambda>0$ flat cosmologies has been found by Pen (1999), while Kayser, Helbig \& Schramm (1997) describe a method to calculate cosmological distances also for inhomogeneous cosmological models. Recently, Hamilton (2001) has also found formulae for the linear growth 
factor and its logarithmic derivative.

On the other hand, the alternative models have not been studied extensively (but after the submission of this paper, the situation has continued to improve; see e.g. Giovi et al. 2000). As simple expressions for the distance-redshift relation are always useful to perform a fast analysis, in this paper I present simple formulae for quintessence cosmological models.

It is assumed that a) the Universe is homogeneous on large-scales, so that Friedmann-Lemaitre equations can be used -in the case of gravitational lensing filled beam (i.e. standard) distances will be adopted-, and b) the equation of state is constant.

In section 2 the basic equations are briefly described, and some exact analytical formulae for the redshift-distance relation are explicitely shown in the trivial cases of one component with $w<0$; it is also shown a formula with elliptic integrals for a realistic matter + quintessence cosmological model with $w=-2 / 3$, which satisfies all the presently available observational constraints.

In section 3 and 4 I discuss the differences among different models with a dark energy component which are expected when applying respectively the AlcockPaczyński test and the statistics of gravitational lensing. The main conclusions are in section 5 .

\section{Redshift-distance relations for models with $w<0$}

\subsection{Basic equations}

For sake of clarity, the basic equations for calculating distances in relativistic cosmology are briefly resumed (e.g. Peebles 1993; Coles \& Lucchin 1996, Peacock 1999; see also Hogg 1999). Assuming that the universe is homogeneous and isotropic universe, we have the Robertson-Walker metric:

$$
d s^{2}=c^{2} d t^{2}-R^{2}(t)\left[d r^{2}+S_{k}(r)\left(d \theta^{2}+\sin ^{2} \theta d \phi^{2}\right)\right]
$$

where $S_{k}(r)=r$ for $k=0, S_{k}(r)=\sin (r)$ for $k=1$ and $S_{k}(r)=\sinh (r)$ for $k=-1$, and from Einstein's field equations the Friedmann equations are obtained:

$$
\begin{gathered}
\left(\frac{\dot{R}}{R}\right)^{2}=H^{2}=\frac{8 \pi G}{3} \sum_{i} \rho_{i}-\frac{k c^{2}}{R^{2}} \\
\frac{\ddot{R}}{R}=-\frac{4 \pi G}{3} \sum_{i} \rho_{i}\left(1+3 w_{i}\right)
\end{gathered}
$$


where $\rho_{i}$ is the energy density of component $i$ and $w_{i}=P_{i} / \rho_{i} c^{2}$ is the corresponding equation of state.

Defining the ratios $\Omega_{i} \equiv \rho_{i} / \rho_{c}$, where $\rho_{c} \equiv 3 H^{2} / 8 \pi G$ and $\Omega_{k} \equiv-k c^{2} /(H R)^{2}$, we can rewrite equation (2) as: $\Omega_{t o t}=\sum_{i} \Omega_{i}=1-\Omega_{k}$.

Moreover, dividing equation (3) by equation (2) we obtain the general expression for Sandage's deceleration parameter $q=-\ddot{R} R / \dot{R}^{2}=\frac{1}{2}\left(1-\Omega_{k}\right)+\frac{3}{2} \sum_{i} \Omega_{i} w_{i}$.

The comoving coordinate $r$ can be written as a function of $\Omega_{i}$ :

$$
r=\sqrt{\left|\Omega_{k}\right|} \int_{0}^{z} \frac{d z^{\prime}}{E\left(z^{\prime}\right)}
$$

where:

$$
E(z)=\sqrt{\Omega_{k}(1+z)^{2}+\sum_{i} \Omega_{i}(1+z)^{3\left(1+w_{i}\right)}}
$$

In what follows I will consider only models with one $w<0$ component and matter $(w=0)$, while radiation will be neglected. In the following, whenever useful, a subscript will indicate the range of values assumed by the equation of state: $w_{Q}$ for values in the range $-1<w<0$, and $w_{V}$ for values less than -1 .

The comoving distance $\prod^{\boxplus}$ is therefore given by:

$$
d_{c}(z)=R_{0} S_{k}(r)=\frac{c}{H_{0}} \frac{1}{\left|\Omega_{k}\right|^{1 / 2}} S_{k}(r)
$$

where, as usual, the subscript 0 indicates the value at the present epoch. The luminosity distance $d_{L}$ is simply given by $d_{L}=d_{c}(1+z)$, while the angular distance is $d_{A}(z)=d_{c} /(1+z)$.

The lookback time is also given by an analogous integral:

$$
t(z)=\frac{1}{H_{0}} \int_{0}^{z} \frac{1}{\left(1+z^{\prime}\right) E\left(z^{\prime}\right)}
$$

Numerical integration (see Press et al. 1992) has been used to check the results obtained with analytical formulae and more generally when no analytical solution was available.

\subsection{One-component models}

Simple analytical expressions can be found only in special cases. Calculations are clearly simplified assuming $w$ constant (a reasonable approximation as far as the equation of state changes slowly with time).

\footnotetext{
${ }^{1} d_{c}(z)$ corresponds to the transverse comoving distance $D_{M}$ as defined by Hogg (1999).
} 
For a flat universe $\left(\Omega_{k}=0\right)$ with only one component, analytical solutions to the integral in equation (4) are very simple. Defining $\alpha \equiv(1+3 w) / 2$, we obtain:

$$
d_{c}(z)= \begin{cases}\frac{c}{H_{0}} \frac{1}{\alpha}\left[1-(1+z)^{-\alpha}\right] & \text { for } \quad w \neq-1 / 3 \\ \frac{c}{H_{0}} \ln (1+z) & \text { for } w=-1 / 3\end{cases}
$$

Other simple solutions can be found by fixing the value of $w$. The values $w=$ $-1 / 3$ and $w=-2 / 3$ are of special interest because they correspond respectively to a frustrated network of cosmic strings and of domain walls (Bucher and Spergel 1999); morevoer, the value $w=-2 / 3$ is consistent with present observations.

For $w=-1 / 3$ we have:

$$
d_{c}(z)=\left\{\begin{array}{llr}
\frac{c}{H_{0}} \frac{\sinh \left(\sqrt{1-\Omega_{Q}} \ln (1+z)\right)}{\sqrt{1-\Omega_{Q}}} & \text { for } & 0<\Omega_{Q}<1 \\
\frac{c}{H_{0}} \ln (1+z) & \text { for } & \Omega_{Q}=1
\end{array}\right.
$$

and for $w=-2 / 3$ :

$$
d_{c}(z)=\left\{\begin{array}{lc}
\frac{c}{H_{0}} \frac{1}{\sqrt{1-\Omega_{Q}}} \ln \frac{z\left(1-\Omega_{Q}\right)+1-\Omega_{Q} / 2+\sqrt{\left[z\left(1-\Omega_{Q}\right)+1\right](1+z)\left(1-\Omega_{Q}\right)}}{\sqrt{1-\Omega_{Q}}+1-\Omega_{Q} / 2} & 0<\Omega_{Q}<1 \\
2 \frac{c}{H_{0}}(\sqrt{1+z}-1) & \Omega_{Q}=1
\end{array}\right.
$$

Figure 1a shows the distance-redshift relation for flat cosmological models with one component having different equations of state, and assuming $H_{0}=65 \mathrm{~km} / \mathrm{s}$ $\mathrm{Mpc}^{-1}$. It is clear that a more negative equation of state implies a larger distance at a given redshift, and that the difference increases with redshift.

\subsection{A formula for the "standard" quintessence model}

Numerical integration of equation (4) is necessary in the general -and realisticcase of a two-component model including both matter and dark energy. In the case of flat cosmological models, the comoving distance of a galaxy at a redshift $z$ can be written using the hypergeometric function ${ }_{2} F_{1}(a, b ; c ; z)$ :

$$
\begin{array}{r}
d_{c}(z)=2 \frac{c}{H_{0}} \frac{1}{\sqrt{1-\Omega_{X}}}\left\{{ }_{2} F_{1}\left[-\frac{1}{6 w}, \frac{1}{2}, 1-\frac{1}{6 w},-\frac{\Omega_{X}}{1-\Omega_{X}}\right]\right. \\
\left.-\frac{1}{\sqrt{1+z}}{ }_{2} F_{1}\left[-\frac{1}{6 w}, \frac{1}{2}, 1-\frac{1}{6 w},-\frac{\Omega_{X}}{1-\Omega_{X}}(1+z)^{3 w}\right]\right\}
\end{array}
$$

where $\Omega_{X}$ refers to the dark energy contribution. The above relation is consistent with the formula found by Torres and Waga (1996) for angular diameter 

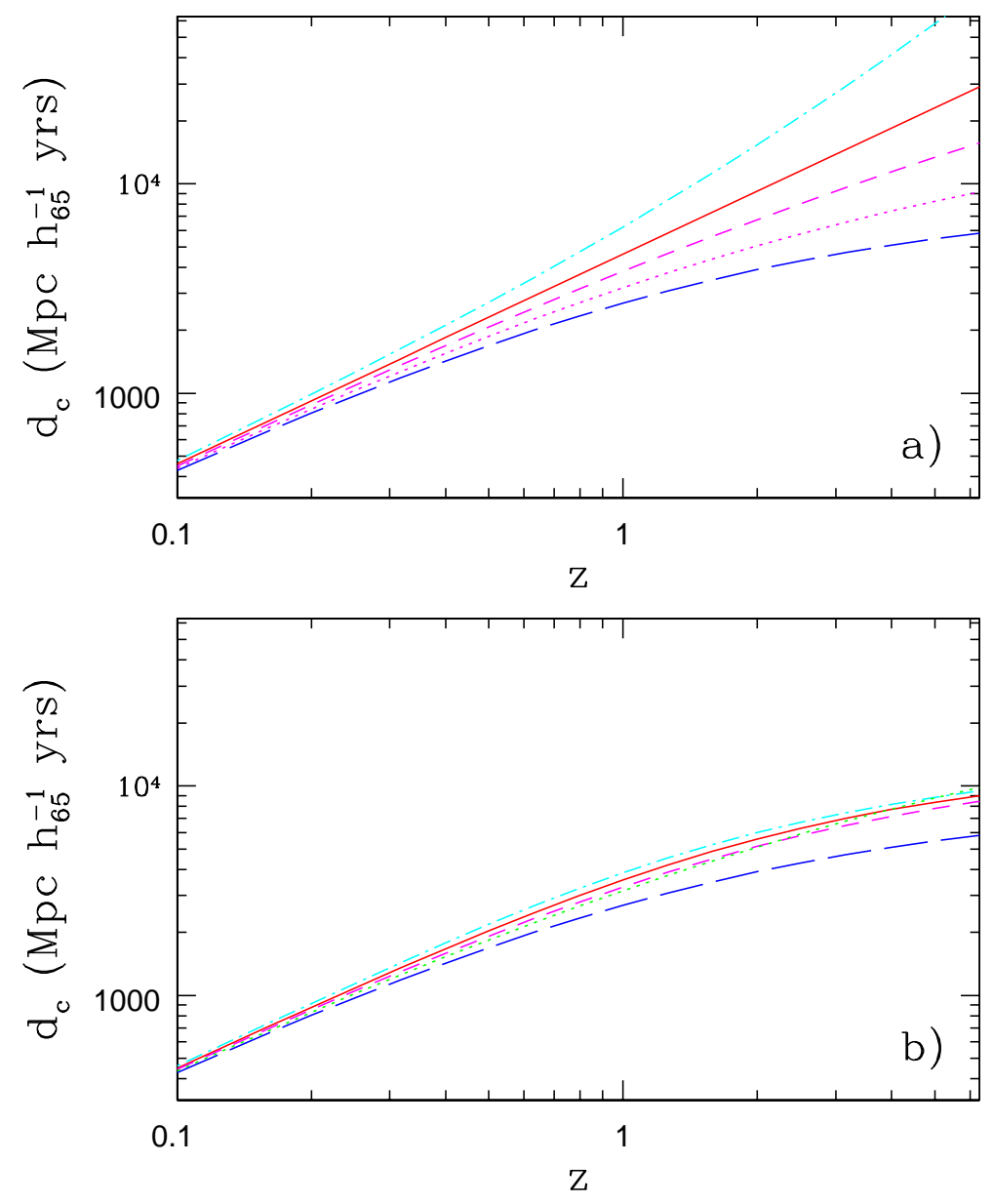

Figure 1: a) Comoving distances for flat cosmological models with one component. Dotted dashed line: $w=-3 / 2$; solid curve: $w=-1$; dashed curve: $w=-2 / 3$; dotted curve: $w=-1 / 3$; long dashed curve: Einstein-de Sitter model. b) Comoving distances for an $\Omega_{M}=0.3$, open model (dotted curve), the Einstein-de Sitter model (long-dashed curve), and 3 flat models, with $\Omega_{M}=0.3$ and an additional negative pressure component $\Omega_{X}=0.7$ with the following equation of state: dashed-dotted curve: $w=-3 / 2$; solid curve: $w=-1$; dashed curve: $w=-2 / 3$. 
distances. Assuming $w=-2 / 3$ it is possible to further simplify the above expression, using only the incomplete elliptic integral of the first kind. A simpler expression can be of practical utility as this equation of state is consistent with the restricted range permitted by present observations (the best parameters for a flat universe are $\Omega_{M}=0.33 \pm 0.05$ and $w_{Q}=0.65 \pm 0.07$ according to Wang et al. 1999).

The formula is the following:

$d_{c}(z)= \begin{cases}C\left[u\left(g(0), \frac{1}{\sqrt{2}}\right)-u\left(g(z), \frac{1}{\sqrt{2}}\right)\right] & 0<\Omega_{Q} \leq 0.5 \\ C\left[u\left(g(z), \frac{1}{\sqrt{2}}\right)-u\left(g(0), \frac{1}{\sqrt{2}}\right)\right] & 0.5<\Omega_{Q}<1, \quad z \leq \sqrt{\frac{\Omega_{Q}}{1-\Omega_{Q}}}-1 \\ C\left[2 u\left(1, \frac{1}{\sqrt{2}}\right)-u\left(g(0), \frac{1}{\sqrt{2}}\right)-u\left(g(z), \frac{1}{\sqrt{2}}\right)\right] & 0.5<\Omega_{Q}<1, \quad z>\sqrt{\frac{\Omega_{Q}}{1-\Omega_{Q}}}-1\end{cases}$

where:

$$
\begin{gathered}
C=\frac{c}{H_{0}} \frac{1}{\left(\Omega_{Q}\left(1-\Omega_{Q}\right)\right)^{1 / 4}} \\
g(z)=2 \frac{\left(\frac{\Omega_{Q}}{1-\Omega_{Q}}\right)^{1 / 4} \sqrt{1+z}}{\sqrt{\frac{\Omega_{Q}}{1-\Omega_{Q}}}+1+z}
\end{gathered}
$$

and

$$
u\left(g, \frac{1}{\sqrt{2}}\right)=\int_{0}^{g} \frac{1}{\sqrt{\left(1-t^{2}\right)\left(1-t^{2} / 2\right)}} d t
$$

is the incomplete elliptic integral of the first kind, which can be easily computed with one of the standard routines available in many mathematical packages. 2 . $u(1,1 / \sqrt{2})=K(1 / \sqrt{2})$ corresponds to the complete elliptic integral of the first kind, and $u(g(0), 1 / \sqrt{2})$ depends only on $\Omega_{Q}$ : when $\Omega_{Q}=0.5, u(g(0), 1 / \sqrt{2})=$ $K(1 / \sqrt{2})$.

Figure $1 \mathrm{~b}$ shows $d_{c}(z)$ for the Einstein-de Sitter model, an open model with $\Omega_{M}=0.3$, and other 3 flat models with $\Omega_{M}=0.3$ and a negative pressure component with $w=-2 / 3(\mathrm{QCDM}), w=-1(\Lambda \mathrm{CDM})$, and $w=-3 / 2(\mathrm{VCDM})$. A synthesis of their main properties is given in table (1). A comparison between figures $1 \mathrm{a}$ and $1 \mathrm{~b}$ shows clearly that the difference between models becomes small in the presence of matter. For example, up to $z \sim 3$ the curve of the open model is very similar to that of the quintessence model with $w=-2 / 3$. 


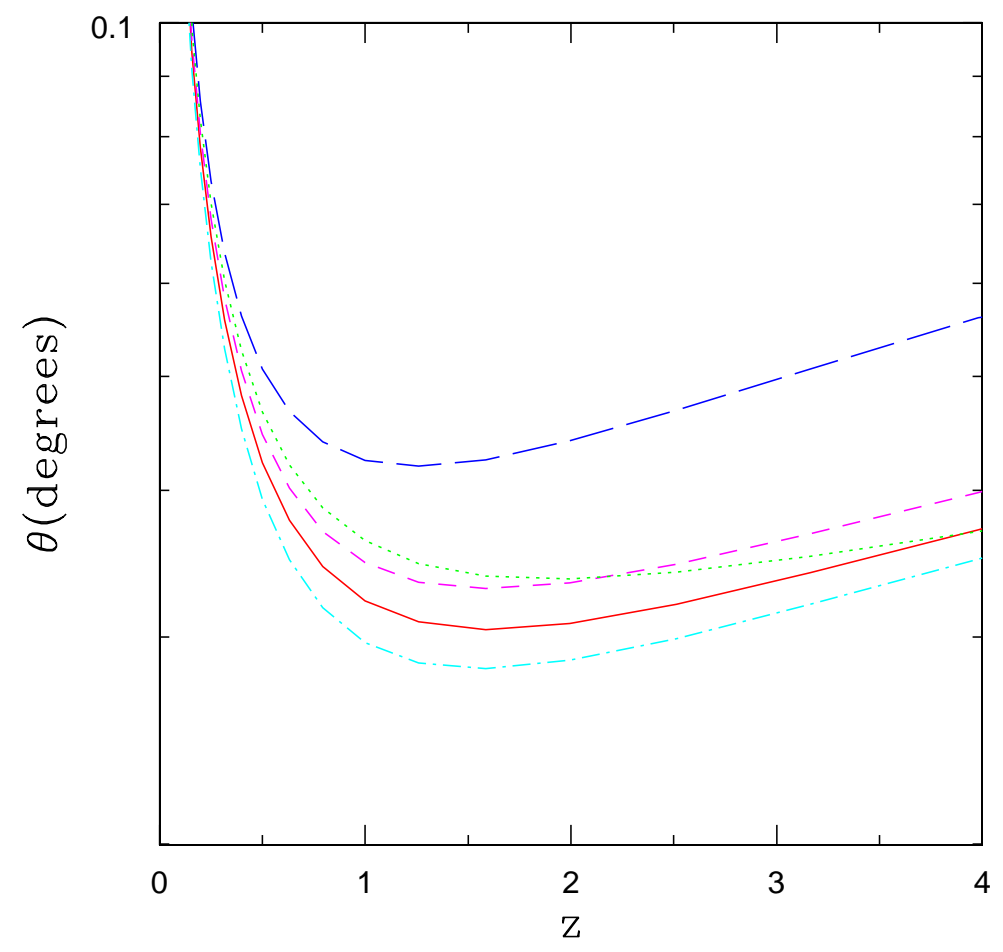

Figure 2: a) Angular diameter corresponding to $1 \mathrm{Mpc}$ (for $H_{0}=65$ $\mathrm{km} / \mathrm{s} / \mathrm{Mpc}$ ) as a function of redshift for the same models as in figure $1 \mathrm{~b}$. Dotted curve: $\Omega_{M}=0.3$, open model; long-dashed curve: Einstein-de Sitter model; 3 flat models, with $\Omega_{M}=0.3$ and an additional negative pressure component $\Omega_{X}=0.7$ with the following equation of state: dashed-dotted curve: $w=-3 / 2$; solid curve: $w=-1$; dashed curve: $w=-2 / 3$. 
Table 1: Properties of different cosmological models

\begin{tabular}{lrrrrr}
\hline & $(1,0,0)$ & $(0.3,0,0)$ & $(0.3,0.7,-2 / 3)$ & $(0.3,0.7,-1)$ & $(0.3,0.7,-3 / 2)$ \\
\hline$q_{0}$ & 0.5 & 0.15 & -0.2 & -0.55 & -1.07 \\
Age $\left(10^{9} \mathrm{~h}_{65}^{-1}\right.$ yrs $)$ & 10.3 & 12.2 & 13.6 & 14.5 & 15.4 \\
$z\left(\theta_{\min }\right)$ & 1.25 & 1.90 & 1.59 & 1.61 & 1.55 \\
$z\left(F_{\max }\right)$ & - & 1.02 & 3.29 & 1.48 & 0.79 \\
$A\left(F_{\max }\right)$ & 1.00 & 1.06 & 1.24 & 1.23 & 1.22 \\
\hline
\end{tabular}

Another interesting quantity is the angular diameter. The angular diameter of an object with physical diameter $D$ is $\theta=\frac{D(1+z)}{d_{c}(z)}=\frac{D(1+z)^{2}}{d_{L}(z)}$. Figure 2 shows the angular diameters corresponding to $1 h_{65}^{-1} \mathrm{Mpc}$ for the same 4 models as in figure 1b. Also in this case the differences between models with negative pressure are not very large, and it is apparent the similarity between the open and standard quintessence models. A detailed discussion of the angular size in the context of quintessence models can be found in Lima \& Alcaniz (2000).

\section{The Alcock-Paczyński test}

Alcock \& Paczyński (1979) showed that, at least in principle, it is possible to detect the presence of the cosmological constant $\Lambda$ in a redshift survey through a geometric test which is independent of galaxy evolution. This method is based on the fact that the spatial distribution of galaxies obtained from the distanceredshift relations valid for an Einstein-de Sitter model is significantly distorted if the universe has a $\Lambda$ component. Therefore a measure of the apparent anisotropy in known isotropic structures would give us the value of the cosmological constant. Phillips (1994) applied this method to the orientation of quasar pairs, without conclusive results. His work has been extended to the quasar correlation function by Popowski et al. (1998). The main problem is that redshift distortions are also induced by peculiar velocities $-\beta$-distortion $\left(\beta \equiv \Omega_{M}^{0.6} / b\right)$ on linear scales and fingers of God on small scales-, but the method might be successfully applied to future redshift surveys, with the simultaneous extraction of $\Lambda$ and $\beta$ from anisotropic power-spectrum data, as discussed by Ballinger, Peacock \& Heavens (1996).

\footnotetext{
${ }^{2}$ For example EllipticF in Maple $\mathrm{V}$ or ellf in Numerical Recipes: notice that ellf requires the quantity $\operatorname{asin}(g)$ instead of $g$.
} 

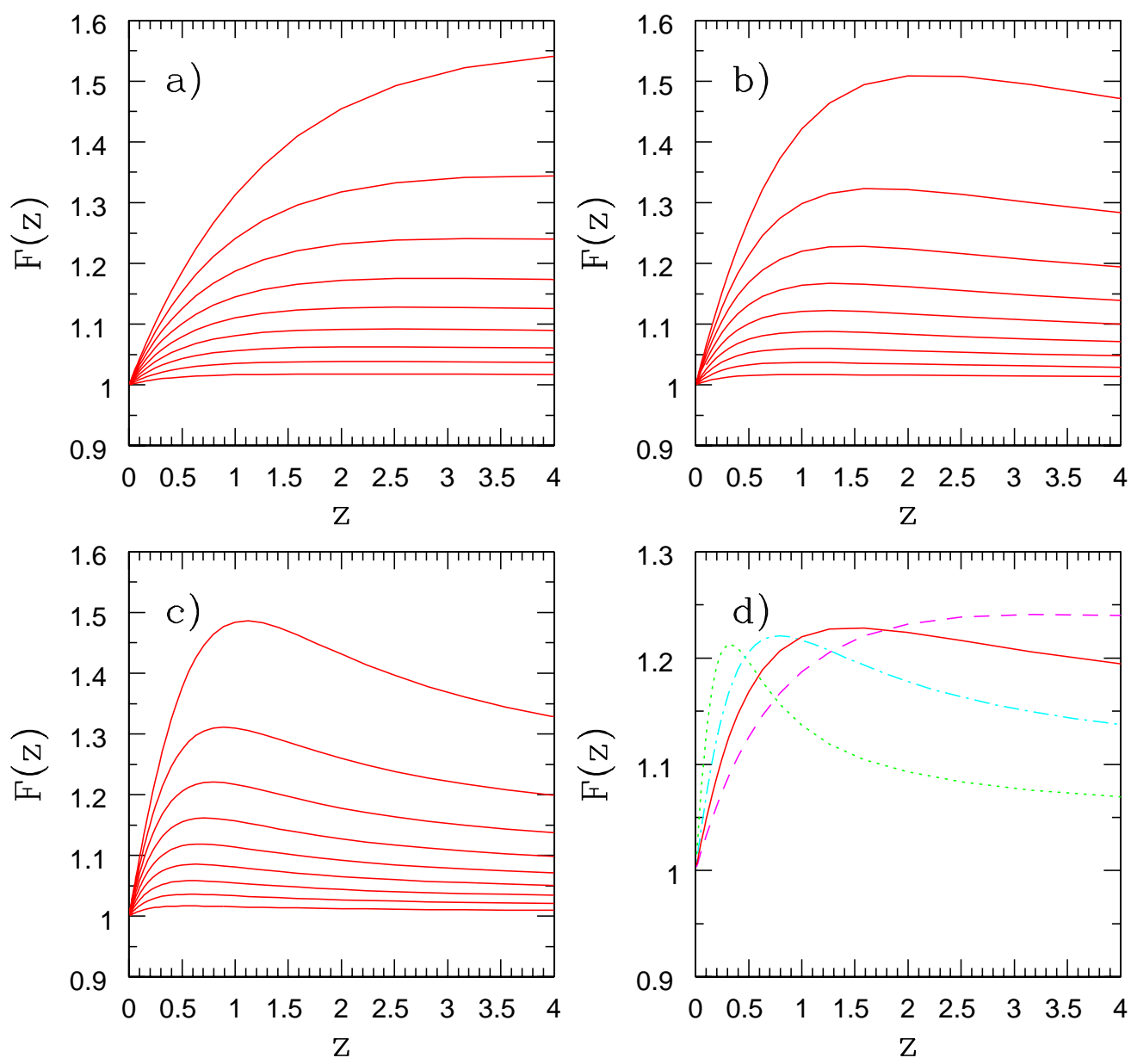

Figure 3: Flattening factor $F(z)$ for different flat models; in figures a-c, the value of $\Omega_{M}$ varies from 0.1 (top curve) to $\Omega_{M}=0.9$ (bottom curve) in steps of 0.1 . a) $\mathrm{QCDM}\left(\Omega_{M}+\Omega_{Q}=1, w_{Q}=-2 / 3\right)$; b) $\Lambda \mathrm{CDM}\left(\Omega_{M}+\Omega_{\Lambda}=1\right)$; c) $\operatorname{VCDM}\left(\Omega_{M}+\Omega_{V}=1, w_{V}=-3 / 2\right)$; d) 4 flat models with $\Omega_{M}=0.3$. Dashed curve: QCDM model with $w_{Q}=-2 / 3$; solid curve: $\Lambda$ CDM model; dashed-dotted curve: VCDM model with $w_{V}=-3 / 2$; dotted curve: VCDM model with $w_{V}=-3$. 
The distortion can be quantified by a flattening factor $F(z)$ relative to the EdS model. In our notation, it is defined as:

$$
F(z) \equiv 2 \frac{c}{H_{0}} \frac{(1+z)^{3 / 2}-(1+z)}{d_{c}(z) E(z)}
$$

$F>1$ indicates a flattening along the line of sight (see Ballinger et al. 1996). Only in special cases $F(z)$ can be expressed analytically: for example, for a flat quintessence-only model $\left(\Omega_{Q}=1\right)$ and $w_{Q}=-2 / 3$, we have $F(z)=\sqrt{1+z}$, to be compared to the $\Omega_{\Lambda}=1$ case, with $F(z)=2(1+z)[\sqrt{(1+z)}-1] / z$.

Note that the formula valid at first order in $z$ given by Ballinger et al. (1996) can be generalized to quintessence models:

$$
F(z)=1+\frac{1}{4}\left[1-\Omega_{M}-\left(1+3 w_{Q}\right) \Omega_{Q}\right] z+O\left(z^{2}\right)
$$

Therefore, when $w_{Q}=-2 / 3$, the test is a direct measure of the difference $\left|\Omega_{M}-\Omega_{Q}\right|$.

In figure $3 \mathrm{a}-\mathrm{c}$ it is shown $F(z)$ for flat models with $w=-2 / 3, w=-1$, and $w=-3 / 2$, while figure 3d shows 4 flat models with $\Omega_{M}=0.3$.

There are three main systematic effects due to the different equation of state which appear clearly in figure $3 \mathrm{~d}$.

With a more negative $w$ we have:

- a) the amplitude of the maximum anisotropy becomes (slightly) smaller;

- b) the maximum of $F(z)$ shifts towards smaller redshifts;

- c) after the maximum, $F(z)$ decays faster.

With a realistic fraction of matter the amplitude of the geometric distortion is not very large, as it amounts to about $20 \%$. After the first analysis of Boomerang results, McGaugh (2000) stressed that a universe with $\Omega_{\Lambda} \sim 1$ and $\Omega_{M}=\Omega_{b}$ would be consistent with results from the observations of distant type Ia supernovae and with the small amplitude of the second peak in the power spectrum of anisotropies in the CMB, if instead of CDM one adopted MOND (Milgrom 1983). In this case, we would expect a larger geometrical distortion, about $30 \%$ at $z=0.5$ and $50 \%$ at $z=1$. However, the more extended analysis of Boomerang results, which has recently identified the first three peaks in the CMB angular power spectrum, shows that a standard flat model with $\Omega_{\Lambda}=0.7$ and $\Omega_{M}=0.3$ is consistent with primordial nucleosynthesis (Netterfield et al. 2001).

An interesting aspect of the Alcock-Paczyński test applied to the VCDM models is that the maximum distortion is shifted towards relatively low redshifts: 
$z_{\max } \sim 0.32$ if $w_{V}=-3$, and even $z_{\max } \sim 0.08$ in an extreme case $w_{V}=-10$. This well-defined maximum at moderate redshifts could be detected in on-going galaxy redshift surveys, or at least it should be possible to fix a lower limit to the equation of state, depending on our ability to disentangle the geometric distortion from the redshift distortion due to peculiar velocities.

\section{Statistics of gravitational lensing}

Gravitational lensing represents an important test of cosmological models, as it is sensitive to the presence of a cosmological constant (Fukugita et al. 1992) or to an equivalent component with negative pressure. Here we simply aim to examine the relative trend for the different models, without going in much detail (among the more recent studies see e.g. Kochanek 1996, Chiba \& Yoshii 1999).

The differential probability $d \tau$ of a line of sight intersecting a lensing galaxy (modelled as a Singular Isothermal Sphere) at redshift $z_{L}$ in the redshift interval $d z_{L}$ is:

$$
d \tau=f \frac{\left(1+z_{L}\right)^{3}}{E\left(z_{L}\right)}\left(\frac{c}{H_{0}}\right)^{-2} \frac{d_{A}\left(0, z_{L}\right) d_{A}\left(z_{L}, z_{S}\right)}{d_{A}\left(0, z_{S}\right)} d z_{L}
$$

For flat models, we have the analytical relation:

$$
\tau\left(z_{S}\right)=\frac{f}{30} d_{c}\left(z_{S}\right)^{3}\left(\frac{c}{H_{0}}\right)^{-3}
$$

where $f$ measures the effectiveness of the lens in producing double images (Turner, Ostriker \& Gott 1984); here its value is estimated assuming a Schechter luminosity function:

$$
f=\frac{16 \pi^{3}}{c H_{0}^{3}} \phi^{*} \sigma^{* 4} \Gamma\left(\alpha+\frac{4}{\gamma}+1\right)
$$

Figure 1 shows the optical depth as a function of the redshift of the source, calculated using the angular diameter distance. With the above relations, it is possible to compare the predictions of gravitational lensing for various cosmological models to observations. Torres \& Waga (1996) have examined the behaviour of a model with $w=-1 / 3$. Here their analysis is extended to include the more popular value $w=-2 / 3$ for quintessence, and the case $w=-3 / 2$ for VCDM. I have used the same catalog, the HST Snapshot Survey (see Maoz et al. 1993), which has detected a number between 3 and 6 of gravitational lensing candidates out of 502 observed quasars. Magnification bias has been taken into account as in Maoz \& 


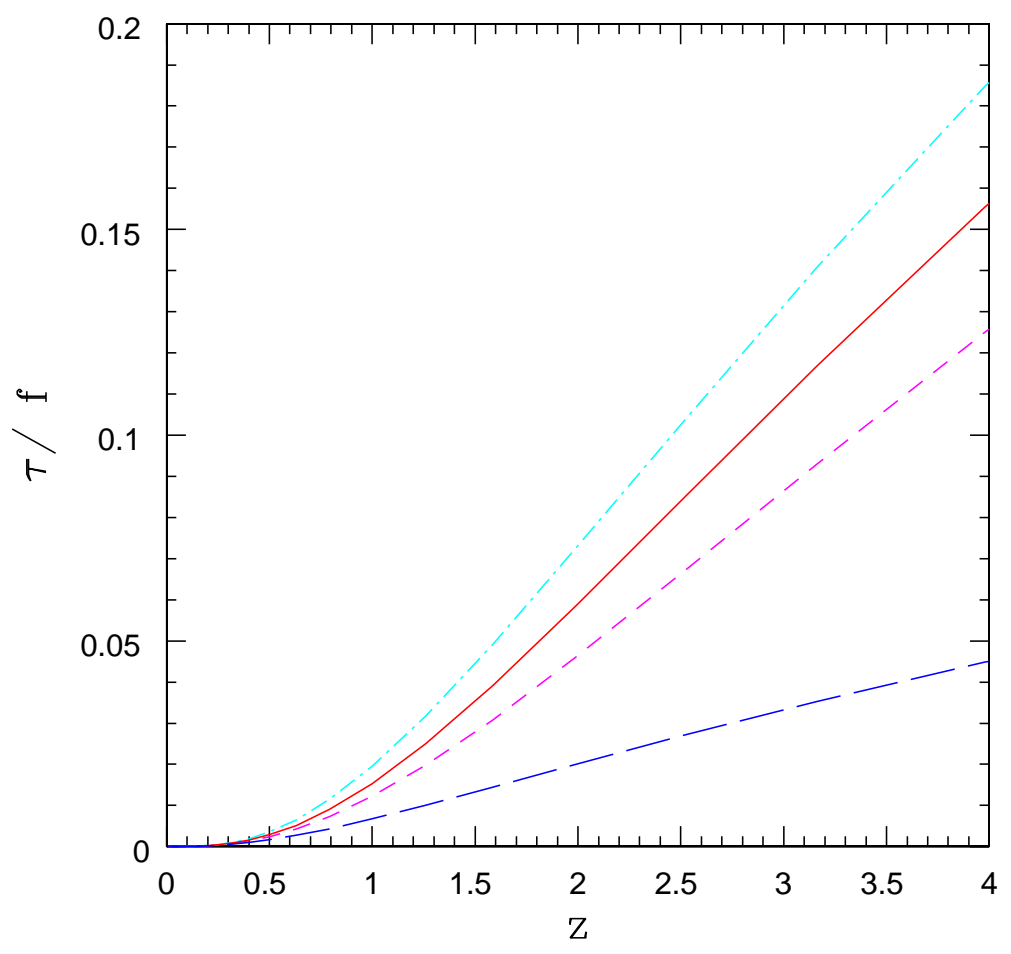

Figure 4: Optical depth for different flat models with $\Omega_{M}=0.3$. From top to bottom: dashed-dotted curve: VCDM model with $w_{V}=-3 / 2$; solid curve: $\Lambda$ model $\left(w_{\Lambda}=-1\right)$; dashed curve: quintessence model with $w_{Q}=-2 / 3$; long-dashed curve: Einstein-de Sitter model. 
Table 2: Predicted number of lensed quasars and corresponding probabilities.

\begin{tabular}{lrrrrr}
\hline & $(1,0,0)$ & $(0.3,0.7,-1)$ & $(0.3,0.7,-2 / 3)$ & $(0.3,0.7,-3 / 2)$ & $(0.5,0.5,-3 / 2)$ \\
\hline Lens no. & 3.7 & 10.8 & 8.6 & 13.5 & 8.0 \\
Prob. & 0.193 & 0.012 & 0.041 & 0.002 & 0.058 \\
\hline
\end{tabular}

$\operatorname{Rix}(1993)$, and I have adopted $\phi^{*}=0.015 \mathrm{~h}^{3} \mathrm{Mpc}^{-3}, \alpha=-1.2, \sigma^{*}=200 \mathrm{~km} / \mathrm{s}$ and $\gamma=4$.

Table 2 resumes the main results, assuming 4 genuine lensing events in the HSTSS. It is well known that $\Lambda>0$ models predict a too large number of lensed quasars, as the optical length increases with $|w|$ (as shown in figure 4; see also Zhu 2000). While the $w_{Q}=-1 / 3$ quintessence model considered by Torres \& Waga (1996) could still be consistent with the data, the same is not true for the $w_{Q}=-2 / 3$ model, and VCDM models perform still worse than the $\Lambda$ model. For VCDM models, more acceptable results can be obtained if the relative contribution of matter is increased. For example, in a fine-tuned VCDM universe with equal contributions of matter and dark energy $\left(\Omega_{M}=0.5\right.$ and $\left.\Omega_{V}=0.5\right)$ the number of lenses becomes comparable to the quintessence $w_{Q}=-2 / 3$ model (see table 2 ). A large matter density results also from the analysis of radio-selected gravitational lenses and optically selected lensed quasars, which gives $\Omega_{M}>0.38$ assuming a flat universe and a nonzero cosmological constant (Falco, Kochanek \& Muñoz 1998).

New surveys should clarify the reasons of this cosmic "discordance" with respect to the increasing observational evidence that the energy density of the universe is dominated by a component with negative pressure: it could be due to systematic errors and uncertainties in the modeling of gravitational lenses (Cheng \& Krauss 2000).

\section{Conclusions}

In this paper various aspects of negative-pressure cosmological models have been analysed:

a) simple redshift-distance formulae (the equivalent of Mattig formulae) have been derived for quintessence cosmological models;

b) it has been shown that the Alcock-Paczyński test is particularly sensitive to $w<-1$ models, where the distortion has a maximum at a relatively low redshift; in the case of a quintessence model with $w=-2 / 3$, this test measures directly the difference $\left|\Omega_{Q}-\Omega_{M}\right|$. 
c) as the more negative is $w$, the larger is the optical depth, a quintessence cosmological model with $w=-2 / 3$ predicts a lower number of lensed quasars -and is therefore more consistent with observations- than the corresponding $\Lambda$ model, while the $w=-3 / 2$ model gives an even larger number of expected lenses, which can be reduced by increasing $\Omega_{M}$.

A more refined analysis is obviously required to test models with negative pressure and their consistency with the full set of observational data (for example, adopting tracking models with a variable equation of state, see Benabed \& Bernardeau 2001).

Oncoming deep redshift surveys, such as the VLT-VIRMOS Deep Survey (Le Fèvre et al. 2001) and DEEP (Davis \& Newman 2001), will sample distances larger than $z \sim 1$, so that it should be possible to obtain constraints to the cosmological parameters which will be complementary to other measurements.

\section{References}

[1] Alcock C., Paczyński B., 1979, Nature 281, 358

[2] Amendola L., 2001, Phys.Rev.Lett. 86, 196

[3] Balbi A., Baccigalupi C., Matarrese S., Perrotta F., Vittorio N., 2001, ApJ 547, L89

[4] Ballinger W.E., Peacock J.A., Heavens A.F., 1996, MNRAS 282, 877

[5] Benabed K., Bernardeau F., 2001 astro-ph/0104371)

[6] Bucher M., Spergel D., 1999, Phys.Rev. D60, 043505

[7] Caldwell R.R., 1999 (astro-ph/9908168)

[8] Caldwell R.R., Dave R., Steinhardt P.J., 1998, Phys.Rev.Lett. 80, 1582

[9] Carroll S.M., 2001, Living Reviews in Relativity 2001-1 (http://www.livingreviews.org, astro-ph/0004075)

[10] Carroll S.M., Press W.H., Turner E.L., 1992, ARA\&A 30, 499

[11] Cheng Y.-C.N., Krauss L.M., 2000, Int.J.Mod.Phys. A15, 697, astro$\mathrm{ph} / 9810393$ )

[12] Chiba M., Yoshii Y., 1999, ApJ 510, 42 
[13] Coles P., Lucchin F., 1996, Cosmology: The Origin and Evolution of Cosmic Structure, John Wiley \& Sons, New York

[14] Davis M., Newman J., 2001, in Proceedings of the ESO/ECF/STSCI Workshop on "Mining the Sky", Garching, in press (astro-ph/0104418)

[15] de Bernardis P. et al., 2000, Nature 404, 955

[16] Efstathiou G., 1999, MNRAS 310, 842

[17] Feige B., 1992, Astron.Nachr. 313, 139

[18] Fukugita M., Futamase T., Kasai M., Turner E.L., 1992, ApJ 393, 3

[19] Giovi F., Occhionero F., Amendola L., 2000, MNRAS, submitted astro $\mathrm{ph} / 0011109$ )

[20] Hamilton A.J.S, 2001, MNRAS, in press astro-ph/0006089)

[21] Hogg D.W., 1999, astro-ph/9905116

[22] Kayser R., Helbig P., Schramm T., 1997, A\&A 318, 680

[23] Kochanek C.S., 1996, ApJ 466, 638

[24] Le Fèvre O., Vettolani G., Maccagni D. et al., 2001, in Proceedings of the ESO/ECF/STSCI Workshop on "Deep Fields", Garching, in press astro$\mathrm{ph} / 0101034$ )

[25] Lima J.A.S., Alcaniz J.S., 2000, A\&A 357, 393

[26] Maoz D., Bahcall J.N., Schneider D.P., Bahcall N.A., Djorgovski S., Doxsey R., Gould A., Kirhakos S., Meylan G., Yanny B., 1993, ApJ 409, 28

[27] Maoz D., Rix H-W, 1993, ApJ 416, 425

[28] Mattig W., 1958, Astron. Nachr. 284, 109

[29] McGaugh S.S., 2000, ApJL, in press (astro-ph/0008188)

[30] Milgrom M., 1983, ApJ. 270, 371

[31] McVittie G.C., 1965, General Relativity and Cosmology, Chapman and Hall LTD, London

[32] Netterfield C.B. et al., 2001, ApJ, submitted (astro-ph/0104460 
[33] Parker L., Raval A., 2001, Phys. Rev. Lett. 86, 749

[34] Peacock J.A., 1999, Cosmological Physics, Cambridge University Press, Cambridge

[35] Peebles P.J.E., 1993, Principles of Physical Cosmology, Princeton University Press, Princeton

[36] Pen Ue-Li, 1999, ApJS 120, 49

[37] Perlmutter S. et al., 1999, ApJ 517, 565

[38] Phillips S., 1994, MNRAS 269, 1077

[39] Podariu S., Ratra B., 2000, ApJ 532, 109

[40] Press W.H., Teukolsky S.A., Vetterling W.T., Flannery B.P., 1992, Numerical Recipes in FORTRAN, 2nd edition, Cambridge University Press http://libwww.lanl.gov/numerical/)

[41] Ratra B., Peebles P.J.E., 1988, Phys.Rev. D 37, 3406

[42] Refsdal S., Stabell R., de Lange F.G., 1967, Mem.RAS 71, 143

[43] Riess A.G. et al., 1998, AJ 114, 722

[44] Riess A.G. et al., 2001, ApJ, in press astro-ph/0104455)

[45] Sahni V., Starobinsky A., 2000, Int.J.Mod.Phys. D9, 373 (astro-ph/9904398)

[46] Schuz A.E., White M., 2001, astro-ph/0104112

[47] Torres L.F.B., Waga I., 1996, MNRAS 279, 712 (astro-ph/0010351)

[48] Turner M.S., 1999, in Type Ia Supernovae: Theory and Cosmology eds. J.Niemeyer \& J.Truran, Cambridge Univ. Press, in press (astro-ph/9904049)

[49] Turner E.L., Ostriker J.P., Gott J.R. III, 1984, ApJ 284, 1

[50] Wang L., Caldwell R.R., Ostriker J.P., Steinhardt P.J., 2000, ApJ 530, 17

[51] Zhu Z.H., 2000, Mod.Phys.Lett. A15, 1023 